\title{
Role of matrix metalloproteinase-7 and apoptosis-associated gene expression levels in the pathogenesis of atrial fibrosis in a Beagle dog model
}

\author{
MIN JIA ${ }^{1}$, ZAI-BO LI ${ }^{1}$, LIN LI ${ }^{1}$, HONG-TAO CHU ${ }^{1}$ and ZHUANG-ZHUANG LI $^{2}$ \\ ${ }^{1}$ Department of Cardiology, Zaozhuang Municipal Hospital; ${ }^{2}$ Department of Cardiology, \\ Shizhong People's Hospital of Zaozhuang, Zaozhuang, Shandong 277102, P.R. China
}

Received July 13, 2016; Accepted April 27, 2017

DOI: $10.3892 / \mathrm{mmr} .2017 .7415$

\begin{abstract}
The aim of the present study was to investigate the potential role of matrix metalloproteinase-7 (MMP-7) and apoptosis-associated genes [TIMP metallopeptidase inhibitor 2(TIMP-2), BCL2 associated X, apoptosis regulator (BAX) and $\mathrm{BCL} 2$, apoptosis regulator (BCL-2)] in the pathogenesis of atrial fibrillation (AF) in a Beagle dog model. A total of 20 adult male Beagle dogs were randomly assigned into the AF group ( $\mathrm{n}=10$; Beagle dogs were treated by Burst stimulation to induce AF) and the control group $(n=10$; healthy Beagle dogs). Echocardiography and Mallory staining were used to determine cardiac function and degree of atrial fibrosis, respectively. Reverse transcription-quantitative polymerase chain reaction and western blotting were performed to determine collagen type 1 (Col I), MMP-7, TIMP-2, BAX and BCL-2 mRNA and protein expression levels. Compared with the control group, the AF group presented increased degree of atrial fibrosis and level of Col I expression, elevated MMP-7 and BAX expression levels, but decreased TIMP-2 and BCL-2 expression levels. Correlation analysis demonstrated that MMP-7 and BAX protein expression levels were negatively correlated with left ventricular ejection fraction (LVEF), but positively correlated with the degree of atrial fibrosis. Negative correlation was observed between TIMP-2 and BCL-2 protein expression levels and degree of atrial fibrosis. In addition, a positive correlation between TIMP-2 and BCL-2 protein expression levels and LEVF was observed. These results demonstrate that MMP-7 and BAX were highly expressed, while TIMP-2 and BCL-2 were downregulated in a Beagle dog model of AF, indicating that MMP-7 and apoptosis-associated genes (TIMP-2, BAX and BCL-2) may be associated with the pathogenesis of AF.
\end{abstract}

Correspondence to: Dr Min Jia, Department of Cardiology, Zaozhuang Municipal Hospital, 41 Longtou Road, Zaozhuang, Shandong 277102, P.R. China

E-mail: zaozhuangjiamin@yeah.net

Key words: matrix metalloproteinase-7, apoptosis-associated gene, atrial fibrosis, atrial fibrillation, Beagle dog model

\section{Introduction}

Atrial fibrillation (AF), the most prevalent arrhythmia, is a risk factor for stroke $(1,2)$. Evidence indicates that subclinical hyperthyroidism is associated with AF, heart failure and coronary disease events (3). In addition, left atrial fibrosis is prominent in patients with AF. Extensive atrial tissue fibrosis identified by delayed enhancement magnetic resonance imaging has been demonstrated to be associated with poor outcomes of AF catheter ablation (4). Atrial fibrosis is hypothesized to be important in the reoccurrence of $\mathrm{AF}$ following cardioversion. It is reported that the expression levels of matrix metalloproteinases (MMPs) and tissue inhibitors of metalloproteinases (TIMPs) may predict AF recurrence following cardioversion and may be considered as candidate novel biomarkers of AF stratification and therapy (5). In addition, the generation of atrial fibrosis is followed by alterations in the expression of apoptosis-associated genes, such as decreased expression of BCL2, apoptosis regulator (BCL-2), and increased expression of BCL-2 associated X, apoptosis regulator (BAX) (6). Therefore, it is considered to be of great clinical significance to investigate the correlation of MMPs and apoptosis-associated gene expression levels with AF.

MMPs are a family of metalloproteinases reported to be associated with numerous biological processes, including ontogenesis morphogenesis, angiogenesis and cell growth (7). MMP-7 and MMP-9, two metalloproteinases in the MMPs family, were identified to be involved in extracellular matrix (ECM) homeostasis and in joint disc remodeling (8). TIMP metallopeptidase inhibitor 2 (TIMP-2), a non-glycosylated protein with a molecular weight of $21 \mathrm{kd}$, is a specific inhibitor of MMPs, which significantly contributes to the inhibition of tumor invasion and metastasis (9). Previous studies investigated the association of MMPs and their inhibitors (TIMPs) with the development and occurrence of AF, and the results indicate that the circulating levels of MMPs and TIMPs are associated with atrial remodeling and prediction of AF recurrence $(5,10)$. BCL-2 functions as a negative regulator in cell apoptosis, which in turn results in the disturbance of homeostatic cell growth. Furthermore, the anti-apoptotic mechanism of BCL-2 involves the inhibition of BAX homo-oligomerization and mitochondrial membrane poration (11). 
The aim of the current study was to investigate the role of MMP-7 and apoptosis-associated gene expression levels in the pathogenesis of AF in a Beagle dog model, in order to provide a meaningful experimental basis for clinical treatment of AF.

\section{Materials and methods}

Ethical approval. The present study was performed in accordance with the approved animal protocols and guidelines established by the Medicine Ethics Review Committee for animal experiments of Zaozhuang Municipal Hospital (Zaozhuang, China).

Animal grouping and establishment of Beagle dog models of $A F$. AF is usually treated with drugs, thus it is relatively difficult to collect surgery tissue samples. Therefore, Beagle dog models of AF were established in the present study. A total of 20 adult male Beagle dogs (age, 1-3 years; weight, 8-10 Kg; purchased from Ya Dong Laboratorial Animal Research Center, Nanjing, China) were selected and randomly divided into the AF group $(n=10)$ and the control group $(n=10)$. Beagles were separately housed at cages at a temperature of $13-16^{\circ} \mathrm{C}$ and humidity of $40-70 \%$, under 12 -h light/dark cycles. Sufficient drinking water was provided for each Beagle dog, and high-quality adult dog food (3-5\% of its weight) was provided twice a day. In the AF group, burst stimulation was used to induce AF. Dogs in the AF group were anaesthetized with ketamine (1 mg/kg; Jiangsu Hengrui medical Co., Ltd', Jiangsu, China; batch no. 20101105). Organon Vecuronium $(0.1 \mathrm{mg} / \mathrm{kg}$; Merck \& Co., Inc., Whitehouse Station, NJ, USA; batch no. 403138) was used to inhibit spontaneous breathing. Tracheal intubation cannulae were used to assist with respiration and Propofol $(200 \mu \mathrm{g} / \mathrm{kg} / \mathrm{min}$; Xi'an Libang Pharmaceutical Co, Ltd., Xi'an, China; batch no. 0711192) was used to maintain anesthesia. The dog hearts were exposed by opening the pericardium through the right fourth intercostal space. Pacemaker electrodes were sutured subcutaneously and placed in the right ventricle. The pacemaker electrode was also sutured in the right atrial appendage as an alternate to induce AF. After closing the chest, the YKE201 pulse generator (Shanghai Yiwu Medical Equipment Co., Ltd, Shanghai, China) was connected and ventricular pacing was set at a frequency of 250 times/min. After surgery, the dogs were clothed with a recovery vest to protect the incision, the pulse generator and the electrode wire. The dogs in the control group underwent the same surgical procedure with the pseudo electrodes stitched. No ventricular pacing was performed in the two groups within 5 weeks of surgery. Five weeks after surgery, Burst stimulation was used to induce AF in the AF group (Pacing cycle length, 100 msec; pacing threshold, four times, 1-10 sec). AF was induced in each Beagle dog in the AF group five times and the mean duration of AF was recorded. If the mean duration was $>15 \mathrm{~min}$, it was recorded as $15 \mathrm{~min}$ and the AF was defined as persistent AF (12). Following measurement of myocardial physiological indices, dogs were sacrificed and the heart was quickly removed. The left and right atrial free walls were isolated. Following the removal of blood and fat tissue, the left and right atrial free walls were quickly frozen with liquid nitrogen and stored at $-80^{\circ} \mathrm{C}$.
Table I. Primer sequences for reverse transcription-quantitative polymerase chain reaction.

\begin{tabular}{ll}
\hline Gene & \multicolumn{1}{c}{ Sequence } \\
\hline MMP-7 & F: 5'-TGGTACCATAATGTCCTGAATG-3' \\
& R:5'-TCGTTATTGGCAGGAAGCACACAAT \\
& GAATT-3' \\
TIMP-2 & F: 5'-GAAACGACATTTATGGCAAC-3' \\
& R: 5'-GATGTTCTTCTCTGTGACCC-3' \\
BAX & F: 5'-TGGCTGGGGAGACACCTGAGC-3' \\
& R: 5'-TCAGCCCATCTTCTTCCAGATG-3' \\
BCL-2 & F: 5'-GCGCTCAGCCCTGTGCCACC-3' \\
& R: 5'-TCATTCAACCAGACATGCAC-3' \\
GAPDH & F: 5'-GGTCACTTGAAGGGTGG-3' \\
& R: 5'-CCATTCGTTGTCGTACCA-3'
\end{tabular}

F, forward; R, reverse; MMP-7, matrix metalloproteinase-7; TIMP-2, TIMP metallopeptidase inhibitor 2; BAX, BCL-2 associated X, apoptosis regulator; BCL-2, BCL2, apoptosis regulator.

Echocardiography. An EnVisor Ultrasound System (iE33, Philips Medical Systems Nederland B.V. Measurements, Best, Netherlands) was used to detect cardiac function parameters, including left atrial diameter (LAD), LAD indexed (LADI) to body surface area, left ventricular end-diastolic diameter (LVEDD), left ventricular septal and posterior end-diastolic wall thickness (PWT of LV) and left ventricular ejection fraction (LVEF) (12).

Atrial specimen collection. After the cardiac function parameters were measured and recorded, the left and right atrial free walls were obtained, blood and adipose tissue were removed, and frozen in liquid nitrogen for preservation at $-80^{\circ} \mathrm{C}$. Preserved atrial tissue samples were used for tissue staining, RNA extraction and protein extraction, as previously described (13).

Mallory's trichrome staining. The preserved atrial tissues were washed with PBS three times, fixed at room temperature for 15-30 min with $4 \%$ paraformaldehyde, embedded in paraffin and sliced in $0.4-\mu \mathrm{m}$ thick sections. Mallory's trichrome staining was performed, as previously described (13). The paraffin sections were dewaxed with xylene and rehydrated. Lugol's iodine salt crystals, alum, hematoxylin dye and acid fuchsin solution $(0.5 \%)$ were added successively for staining. Staining was performed at room temperature for 1-5 min and full washing with water was performed after each stain; when the fiber was colorless, washing with distilled water was performed. Subsequent to Mallory's trichrome staining, the sections were stained for 20 min with Aniline Blue solution and alcohol (95\%) was added for hydration. Finally, anhydrous ethanol was used for dehydration. The tissue block was cleared in xylene and mounted. After staining, the collagen fiber was dark blue and the muscle tissues were bright orange. Images were obtained using an optical microscope and the fibrosis rate was calculated, as previously described (14). 
Table II. Comparison of cardiac function parameters between the AF and control groups.

\begin{tabular}{lcrr}
\hline Parameter & Control group $(\mathrm{n}=10)$ & AF group $(\mathrm{n}=10)$ & P-value \\
\hline LAD $(\mathrm{mm})$ & $31.61 \pm 4.19$ & $23.91 \pm 3.36$ & $<0.001$ \\
LADI $\left(\mathrm{cm} / \mathrm{m}^{2}\right)$ & $2.47 \pm 0.14$ & $3.72 \pm 0.38$ & $<0.001$ \\
LVEDD $(\mathrm{mm})$ & $1.05 \pm 0.10$ & $0.98 \pm 0.11$ & 0.156 \\
LVEF $(\%)$ & $57.77 \pm 4.39$ & $51.57 \pm 5.31$ & 0.011 \\
LVPWT $(\mathrm{mm})$ & $7.35 \pm 0.85$ & $7.31 \pm 1.33$ & 0.937 \\
\hline
\end{tabular}

AF, atrial fibrillation; LAD, left atrial diameter; LADI, left atrial diameter indexed to body surface area; LVEDD, left ventricular end-diastolic diameter; LVEF, left ventricular ejection fraction; LVPWT, left ventricular septal and posterior end-diastolic wall thickness.

$R N A$ extraction and reverse transcription- quantitative polymerase chain reaction $(R T-q P C R)$. The preserved atrial tissues were washed with PBS three times. Total RNA was extracted using RNAiso Plus kit (Takara Biotechnology Co., Ltd., Dalian, China) according to the manufacturer's protocol, and RT was performed using the Prime Script RT reagent kit (Takara Bio, Inc., Otsu, Japan), according to the manufacturer's protocol. qPCR was performed on a StepOnePlus PCR instrument (Applied Biosystems; Thermo Fisher Scientific, Inc., Waltham, MA, USA), as previously described (13). The primer sequences that were used in the present study are presented in Table I. The qPCR reaction included the following: $1.6 \mu \mathrm{l}$ cDNA solution, $5 \mu 12 X$ SYBR GREEN Taq PCR mix (Takara Bio, Inc.), $0.2 \mu \mathrm{l}$ of each forward and reverse primer $(10 \mu \mathrm{M})$

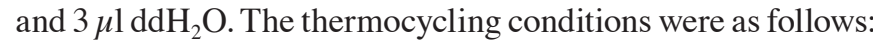
Pre-denaturation at $95^{\circ} \mathrm{C}$ for $5 \mathrm{~min}$, followed by 60 cycles at $95^{\circ} \mathrm{C}$ for $10 \mathrm{sec}$, at $58^{\circ} \mathrm{C}$ for $10 \mathrm{sec}$ and at $72^{\circ} \mathrm{C}$ for $10 \mathrm{sec}$, with a final extension step at $72^{\circ} \mathrm{C}$ for $10 \mathrm{~min}$. MMP-7 (Gene ID 489432), TIMP-2 (Gene ID 403633), BAX (Gene ID 403523) and BCL-2 (Gene ID 403416) were detected. GAPDH (Gene ID 403755) served as the internal reference. Melting curve was applied to assess the reliability of the PCR products. Relative gene expression was calculated according to the comparative Cq method (15) and normalized to GAPDH.

Western blot analysis. The preserved atrial tissues were cleaned with distilled water, and total proteins were extracted using radioimmunoprecipitation assay lysis buffer (Gibco; Thermo Fisher Scientific Inc.) with protease inhibitors (Sigma-Aldrich; Merck KGaA, Darmstadt, Germany) on ice for 5-15 min. Centrifugation was performed at $4^{\circ} \mathrm{C}$ at a speed of $12,000 \mathrm{x} \mathrm{g}$ for $10 \mathrm{~min}$. The supernatants were collected and protein concentration was detected using a bicinchoninic acid protein assay (Bio-Rad Laboratories, Inc., Hercules, CA, USA). Equal amounts of extracted protein samples $(20 \mu \mathrm{g})$ were separated by $8 \%$ SDS-PAGE and transferred onto nitrocellulose membranes. Membranes were probed with the following primary antibodies at $4^{\circ} \mathrm{C}$ overnight: Anti-rabbit immunoglobulin (Ig) G Fc antibody (RMG02; cat. no. ab190492; 1:500; Abcam, Cambridge, MA, USA), anti-MMP-7 (cat no. ab189277; 1:500; Abcam), anti-Col I (cat no. ab90395; 1:20; Abcam), anti-TIMP-2 (cat no. ab180630; 1:500; Abcam), anti-BCL-2 (cat no. ab59348; 1:500; Abcam) and anti-BAX (cat no. ab32503; 1:1,000; Abcam). Subsequently, they were incubated with horseradish peroxidase-conjugated
Table III. Correlation of the expression levels of MMP-7 and apoptosis-associated genes, TIMP-2, BAX and BCL-2 with LVEF and the degree of cardiac fibrosis between the AF and control groups.

\begin{tabular}{|c|c|c|c|c|}
\hline \multirow[b]{2}{*}{ Gene } & \multicolumn{2}{|c|}{ LVEF } & \multicolumn{2}{|c|}{$\begin{array}{c}\text { Degree } \\
\text { of cardiac fibrosis }\end{array}$} \\
\hline & $\mathrm{r}$ & P-value & $\mathrm{r}$ & P-value \\
\hline MMP-7 & -0.92 & $<0.001$ & 0.88 & 0.001 \\
\hline TIMP-2 & 0.96 & $<0.001$ & -0.92 & $<0.001$ \\
\hline BAX & -0.96 & $<0.001$ & 0.92 & $<0.001$ \\
\hline BCL-2 & 0.97 & 0.002 & -0.95 & $<0.001$ \\
\hline
\end{tabular}

LVEF, left ventricular rejection fraction; MMP-7, matrix metalloproteinase-7; TIMP-2, TIMP metallopeptidase inhibitor 2; BAX, BCL-2 associated $\mathrm{X}$, apoptosis regulator; BCL-2, BCL2, apoptosis regulator.

goat anti-rabbit IgG (cat. no. ab6721; 1:5,000; Abcam) at room temperature for $30 \mathrm{~min}$. Then Horseradish Peroxidase (HRP) (Bio-Rad Laboratories, Inc.) was added for color rendering. Protein bands were visualized using enhanced chemiluminescence. Blots were semi-quantified by densitometry using Image Quant 350 and Image Quant TL software version 1 (GE Healthcare Life Sciences, Little Chalfont, UK). $\beta$-actin served as the internal control (13).

Statistical analysis. Statistical analyses were performed sing SPSS software version 20.0 (IBM Corp., Armonk, NY, USA). Data are expressed as the means \pm standard deviation. The statistical significance of the differences between groups was assessed unpaired Student's t-test. $\mathrm{P}<0.05$ was considered to indicate a statistically significant difference. In addition, the Pearson correlation test was used for correlation analysis with $0.8<\mathrm{r}<1.0$ considered as a strong correlation.

\section{Results}

Comparison of cardiac function parameters between the $A F$ and control groups. LVEF and LAD in the control group were significantly higher than those in the AF group $(\mathrm{P}<0.05)$; while LADI in the control group was significantly lower than 
A
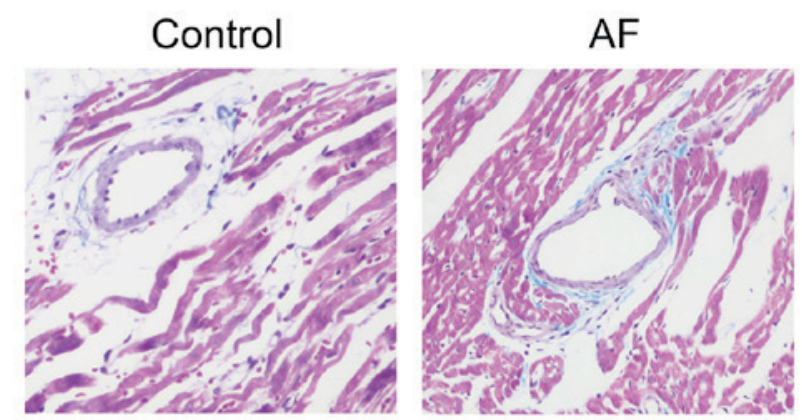

C

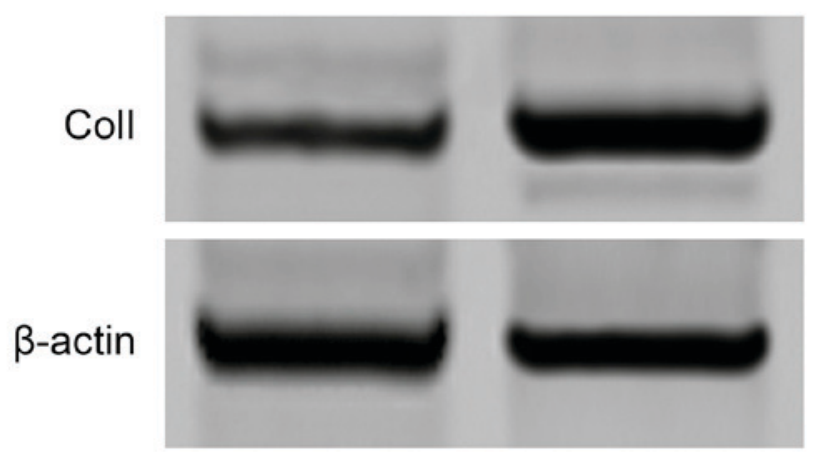

B

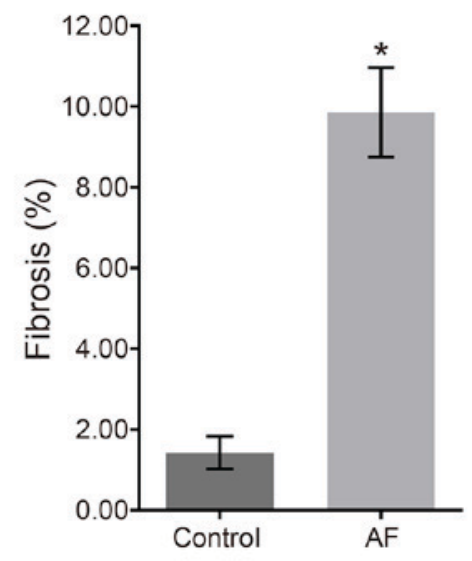

D

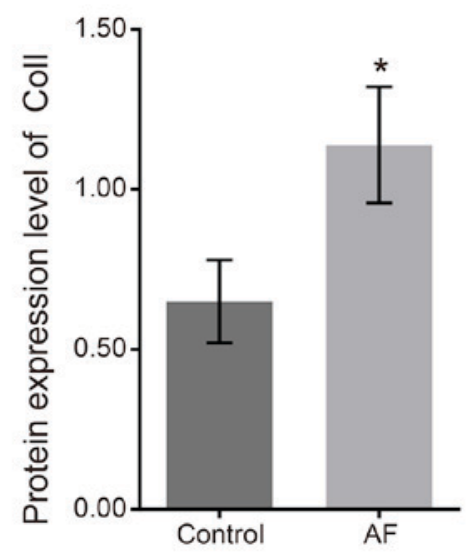

Figure 1. Comparison of cardiac fibrosis between the AF and control groups. (A) Mallory's trichrome staining map of cardiac fibrosis, the fibrotic tissues were stained a blue-violet color (B) Percentage of fibrotic tissue. (C) Western blot results of fibrosis factor, Col I protein expression. (D) Quantification of protein expression levels obtained by western blotting. Data are expressed as the mean \pm standard deviation. ${ }^{*} \mathrm{P}<0.05$ vs. the control group. AF, atrial fibrillation group; Col I, collagen type 1 .

that in the AF group $(\mathrm{P}<0.05)$. No significant difference was identified in LVEDD and LVPWT between the two groups (P>0.05; Table II). The cardiac function of the AF group was demonstrated to be significantly lower than that of the control group, indicating the successful establishment of an AF model.

Comparison of cardiac fibroses between the AF and control groups. The Mallory's trichrome staining results of atrial fibrosis (Fig. 1A) demonstrated that the degree of atrial fibrosis in the control group was significantly lower than that in the AF group. In the AF group, the fibrous tissue samples in the left atrial myocytes in the myocardial tissue were compact and intact, meanwhile muscle bundles were separated by connective tissues, which was characterized by over accumulation of collagen and deposition in connective tissues. As shown in Fig. 1B, the proportion of fibrotic tissue in the control group $(1.43 \pm 0.40 \%)$ was significantly lower than that in the AF group $(9.86 \pm 1.11 \%$; $\mathrm{P}<0.05)$. The western blotting result of atrial fibrosis factor $\mathrm{Col}$ I (Fig. 1C) demonstrated that the relative expression of Col I protein in the control group was $0.65 \pm 0.13$, which was significantly lower than that in the AF group $(1.14 \pm 0.18 ; \mathrm{P}<0.05)$. The results of protein expression (Fig. 1D) indicated that the degree of cardiac fibrosis was increased following AF.
Comparisons of the mRNA expression levels of MMP-7 and other apoptosis-associated genes between the AF and control groups. GAPDH mRNA expression in the control group was set as 1. Compared with the control group, MMP-7 and BAX mRNA expression levels in the AF group were elevated $(\mathrm{P}<0.05)$, while BCL-2 and TIMP-2 mRNA expression levels were decreased $(\mathrm{P}<0.05$; Fig. 2). The results indicate that the elevated mRNA expression levels of MMP-7 and BAX, and the decreased mRNA expression levels of TIMP-2 and BCL-2 may lead to AF.

Comparisons of the protein expression levels of MMP-7 and other apoptosis-associated genes between the AF and control groups. The protein level of the relative reference, $\beta$-actin in the control group was set as 1. Compared with the control group, protein expression levels of MMP-7 and BAX were elevated $(\mathrm{P}<0.05)$, while the protein expression levels of TIMP-2 and BCL-2 were decreased in the AF group $(\mathrm{P}<0.05)$. The results demonstrated that elevated expression levels of MMP-7 and BAX, and decreased expression levels of BCL-2 and TIMP-2 may be associated with AF occurrence (Fig. 3).

Correlation of the protein expression levels of MMP-7 and other apoptosis-associated genes with the cardiac function 
A

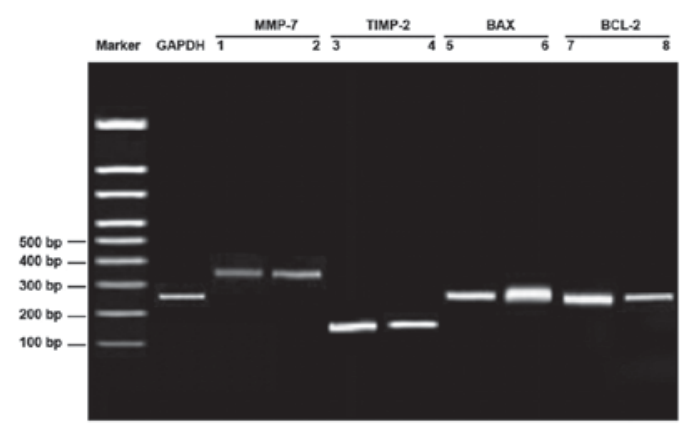

B

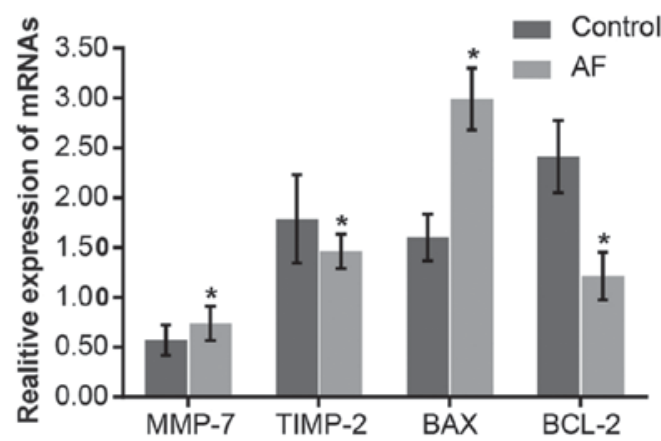

Figure 2. Comparison of the mRNA expression levels of MMP-7 and other apoptosis-associated genes between the AF and control groups. (A) Reverse transcription-quantitative polymerase chain reaction of MMP-7 and other apoptosis-associate genes. The expression levels of the control group are presented in bands 1, 3, 5 and 7, and the expression levels of the AF group are presented in bands 2, 4, 6 and 8. (B) mRNA expression levels of MMP-7 and other apoptosis-associated genes. Data are expressed as the mean \pm standard deviation. " $\mathrm{P}<0.05$ vs. the control group. AF, atrial fibrillation; MMP-7, matrix metalloproteinase-7; TIMP-2, TIMP metallopeptidase inhibitor 2; BAX, BCL-2 associated X, apoptosis regulator; BCL-2, BCL2, apoptosis regulator.

A

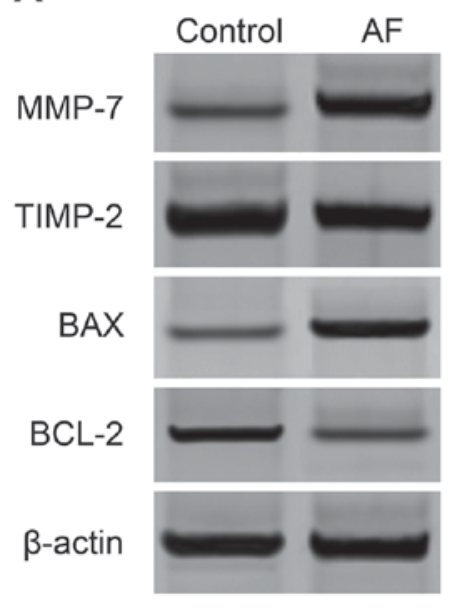

B

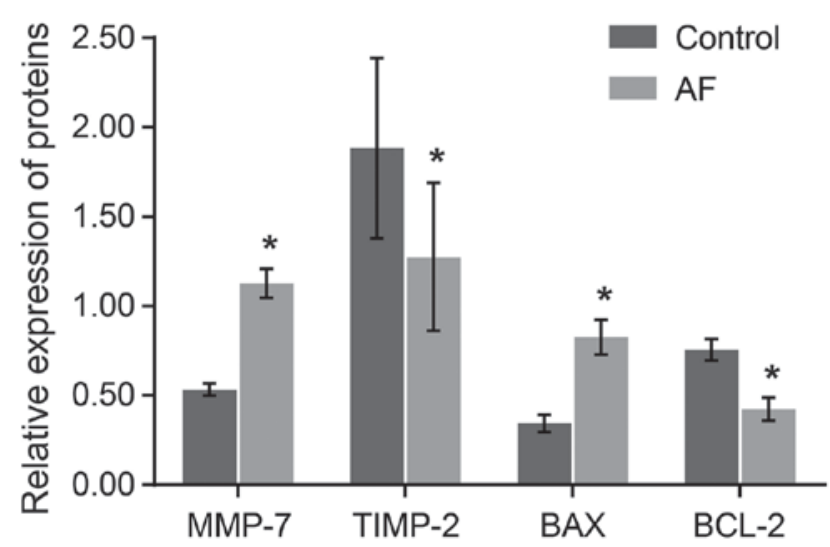

Figure 3. Comparison of the protein expression levels of MMP-7 and other apoptosis-associated genes between the AF and control groups. (A) Western blot results and (B) relative protein expression levels of MMP-7 and other apoptosis-associated genes. Data are expressed as the mean \pm standard deviation. ${ }^{*} \mathrm{P}<0.05$ vs. the control group. AF, atrial fibrillation group; MMP-7, matrix metalloproteinase-7; TIMP-2, TIMP metallopeptidase inhibitor 2; BAX, BCL-2 associated $\mathrm{X}$, apoptosis regulator; BCL-2, BCL2, apoptosis regulator.

parameters of $A F$. Pearson correlation analysis was used to analyze the correlation between MMP-7 together with apoptosis-associated genes and the cardiac function parameters of AF. According to the correlation analysis, MMP-7 and BAX expression levels were negatively correlated with LVEF ( $\mathrm{r}=-0.92 ; \mathrm{r}=-0.96 ; \mathrm{P}<0.001)$, and TIMP-2 and BCL-2 expression levels were positively correlated with LVEF $(\mathrm{r}=0.96 ; \mathrm{P}<0.001 ; \mathrm{r}=0.97 ; \mathrm{P}=0.002)$. In addition, MMP-7 and BAX expression levels were positively correlated with the degree of cardiac fibrosis $(\mathrm{r}=0.88 ; \mathrm{P}=0.001 ; \mathrm{r}=0.92 ; \mathrm{P}<0.001)$, while TIMP-2 and BCL-2 expression levels were negatively correlated with the degree of cardiac fibrosis $(r=-0.92 ; r=-0.95$; $\mathrm{P}<0.001$; Table III).

\section{Discussion}

By detecting the mRNA and protein expression levels of MMP-7 and apoptosis-associated genes (TIMP-2, BAX and
BCL-2), the major finding of the current study is that MMP-7 and apoptosis-associated genes (TIMP-2, BAX and BCL-2) may serve as indicators of $\mathrm{AF}$, and provide direction for the prognosis and treatment of AF.

According to the experimental results, the expression level of MMP-7 in the AF group was increased when compared with that of the control group, which indicated that the mRNA expression levels of MMP-7 and other apoptosis-associated genes are likely to be associated with AF. Increasing evidence indicates that MMPs are important in normal embryonic development, tissue remodeling, and pathological and physiological processes occurring in the human body (16). Regulating the expression and activity of MMPs and TIMPs may represent an important clinical treatment strategy to prevent atrial remodeling (17). A previous study indicated that MMPs and TIMPs are essential for cardiac ECM remodeling (18). ECM is a dynamic structure, whose physiological turnover is mediated by MMPs and their TIMPs. In addition to providing structural 
support, the ECM also function as an extracellular reservoir for multiple growth factors and cytokines (19). However, MMPs are crucial in the collagen metabolism process of atrial tissue via gene transcription, protein expression and activity elevation, causing excessive degradation of normal collagen and abnormal myocardial cell gaps in the ECM. The the gap is subsequently filled with other fibrous tissues and fibrosis gradually increases (20). TIMPs bind to the active site of the MMPs in a stoichiometric 1:1 molar ratio, thereby blocking access to the ECM substrates, and also stimulate the proliferation and differentiation of fibroblasts in cardiac tissue damage sites, thereby promoting synthesis of the ECM $(21,22)$. In the infarcted myocardium, activation of the inflammatory cascade clears the wound of dead cells, whereas stimulating matrix degradation and chamber dilation contributes to the development of heart failure (23).

The current study demonstrated that the low expression level of BCL-2 and the high expression level of BAX were associated with the occurrence and development of AF. Previous studies indicate that there are two phylogenetically and structurally distinct groups of proteins regulating the stress-induced intrinsic apoptosis, i.e., the programmed disassembly of cells, among which BCL-2 is an anti-apoptosis regulator while BAX is a pro-apoptosis protein (24). As BAX and BCL-2 exert opposite regulatory effects on cell apoptosis, it is considered that there may be a balance between BAX and BCL-2, which co-regulates the apoptosis of cells. A previous study found that in cases of atrial aging or AF, the level of BCL-2 expression in the left atrial myocardial tissue was downregulated, while the level of BAX expression was elevated (13). In addition, it was reported that apoptosis was significantly elevated, as indicated by the increasing BAX/BCL-2 ratio, after patients with gastric adenocarcinoma received crocin treatment (25). Furthermore, a recent study indicated that MMP may be involved in the occurrence and development of AF via upregulating BAX expression levels and downregulating BCL-2 expression levels, which is consistent with the results of the current study (26). However, a limitation of the present study was the relatively small number of subjects; thus, future studies with a larger sample are required to verify the present results.

In conclusion, the present study provides evidence that MMP-7 and apoptosis-associated gene expression levels are correlated with the cardiac function parameters of AF. However, the underlying mechanisms of these genes in the occurrence and development of AF remains unknown. Future investigations will focus on the correlation analysis between the epidemiology of cardiovascular disease and associated gene expression, with the aim of improving knowledge of gene therapy for AF, as well as myocardial infraction, dilated cardiomyopathy and heart failure.

\section{Acknowledgements}

The authors would like to acknowledge the helpful comments received from our reviewers for this study.

\section{References}

1. Gu J, Hu W and Liu X: The value of magnetic resonance imaging in catheter ablation of atrial fibrillation. Clin Cardiol 38: 190-194, 2015.
2. Gandolfo C, Balestrino M, Bruno C, Finocchi C and Reale N: Validation of a simple method for atrial fibrillation screening in patients with stroke. Neurol Sci 36: 1675-1678, 2015.

3. Carpi A, Cini G, Russo M, Antonelli A, Gaudio C, Galetta F, Franzoni F and Rossi G: Subclinical hyperthyroidism and cardiovascular manifestations: A reevaluation of the association. Intern Emerg Med 8 (Suppl 1): S75-S77, 2013.

4. Marrouche NF, Wilber D, Hindricks G, Jais P, Akoum N Marchlinski F, Kholmovski E, Burgon N, Hu N, Mont L, et al: Association of atrial tissue fibrosis identified by delayed enhancement MRI and atrial fibrillation catheter ablation: The DECAAF study. JAMA 311: 498-506, 2014.

5. Mukherjee R, Akar JG, Wharton JM, Adams DK, McClure CD, Stroud RE, Rice AD, DeSantis SM, Spinale FG and Gold MR: Plasma profiles of matrix metalloproteinases and tissue inhibitors of the metalloproteinases predict recurrence of atrial fibrillation following cardioversion. J Cardiovasc Transl Res 6: 528-535, 2013.

6. Wang Y, Zhang H, Chai F, Liu X and Berk M: The effects of escitalopram on myocardial apoptosis and the expression of Bax and Bcl-2 during myocardial ischemia/reperfusion in a model of rats with depression. BMC Psychiatry 14: 349, 2014.

7. Papazafiropoulou A and Tentolouris N: Matrix metalloproteinases and cardiovascular diseases. Hippokratia 13: 76-82, 2009.

8. Loreto C, Leonardi R, Musumeci G, Pannone G and Castorina S: An ex vivo study on immunohistochemical localization of MMP-7 and MMP-9 in temporomandibular joint discs with internal derangement. Eur J Histochem 57: e12, 2013.

9. O'Grady A,Dunne C, O'Kelly P, Murphy GM, Leader M and Kay E: Differential expression of matrix metalloproteinase (MMP)-2, MMP-9 and tissue inhibitor of metalloproteinase (TIMP)-1 and TIMP-2 in non-melanoma skin cancer: Implications for tumour progression. Histopathology 51: 793-804, 2007.

10. Chen CL, Huang SK, Lin JL, Lai LP, Lai SC, Liu CW, Chen WC, Wen $\mathrm{CH}$ and Lin CS: Upregulation of matrix metalloproteinase-9 and tissue inhibitors of metalloproteinases in rapid atrial pacing-induced atrial fibrillation. J Mol Cell Cardiol 45: 742-753, 2008.

11. Barclay LA, Wales TE, Garner TP, Wachter F, Lee S, Guerra RM, Stewart ML, Braun CR, Bird GH, Gavathiotis E, et al: Inhibition of Pro-apoptotic BAX by a noncanonical interaction mechanism. Mol Cell 57: 873-886, 2015.

12. Lewkowicz J, Knapp M, Tankiewicz-Kwedlo A, Sawicki R, Kamińska M, Waszkiewicz E and Musiał WJ: MMP-9 in atrial remodeling in patients with atrial fibrillation. Ann Cardiol Angeiol (Paris) 64: 285-291, 2015.

13. Xu GJ, Gan TY, Tang BP, Chen ZH, Mahemuti A, Jiang T, Song JG, Guo X, Li YD, Miao HJ, et al: Accelerated fibrosis and apoptosis with ageing and in atrial fibrillation: Adaptive responses with maladaptive consequences. Exp Ther Med 5: 723-729, 2013.

14. Myers RP, Patel K, Pianko S, Poynard T and McHutchison JG: The rate of fibrosis progression is an independent predictor of the response to antiviral therapy in chronic hepatitis C. J Viral Hepat 10: 16-22, 2003.

15. Livak KJ and Schmittgen TD: Analysis of relative gene expression data using real-time quantitative PCR and the 2(-delta delta C(T)) method. Methods 25: 402-408, 2001.

16. Tesfaigzi Y, Myers OB, Stidley CA, Schwalm K, Picchi M, Crowell RE, Gilliland FD and Belinsky SA: Genotypes in matrix metalloproteinase 9 are a risk factor for COPD. Int $\mathbf{J}$ Chron Obstruct Pulmon Dis 1: 267-278, 2006.

17. Wang W, Zhang HT and Yang XL: Effect of matrix metalloproteinase and their inhibitors on atrial myocardial structural remodeling. J Cardiovasc Med (Hagerstown) 14: 265-269, 2013.

18. Kalogeropoulos AS, Tsiodras S, Rigopoulos AG, Sakadakis EA, Triantafyllis A, Kremastinos DT and Rizos I: Novel association patterns of cardiac remodeling markers in patients with essential hypertension and atrial fibrillation. BMC Cardiovasc Disord 11: 77, 2011.

19. Takawale A, Sakamuri SS and Kassiri Z: Extracellular matrix communication and turnover in cardiac physiology and pathology. Compr Physiol 5: 687-719, 2015.

20. Spinale FG: Myocardial matrix remodeling and the matrix metalloproteinases: Influence on cardiac form and function. Physiol Rev 87: 1285-1342, 2007.

21. Camelliti P, Borg TK and Kohl P: Structural and functional characterisation of cardiac fibroblasts. Cardiovasc Res 65: 40-51, 2005. 
22. Vanhoutte D, Schellings M, Pinto Y and Heymans S: Relevance of matrix metalloproteinases and their inhibitors after myocardial infarction: A temporal and spatial window. Cardiovasc Res 69: 604-613, 2006

23. Saxena A, Chen W, Su Y, Rai V, Uche OU, Li N and Frangogiannis NG: IL-1 induces proinflammatory leukocyte infiltration and regulates fibroblast phenotype in the infarcted myocardium. J Immunol 191: 4838-4848, 2013.

24. Kvansakul M and Hinds MG: The Bcl-2 family: Structures, interactions and targets for drug discovery. Apoptosis 20 : 136-150, 2015.
25. Hoshyar R, Bathaie SZ and Sadeghizadeh M: Crocin triggers the apoptosis through increasing the $\mathrm{Bax} / \mathrm{Bcl}-2$ ratio and caspase activation in human gastric adenocarcinoma, AGS, cells. DNA Cell Biol 32: 50-57, 2013

26. Diao SL, Xu HP, Zhang B, Ma BX and Liu XL: Associations of MMP-2, BAX, and BCL-2 mRNA and protein expressions with development of atrial fibrillation. Med Sci Monit 22: 1497-1507, 2016. 